\title{
On Waring's Problem:
}

$$
\mathrm{g}(4)<20
$$

\section{R. BALASUBRAMANIAN}

\$1. In [1], I proved that every integer is a sum of atmost 21 biquadrates. The object of this paper is to prove a refinement namely

Theorem : Every positive integer is a sum of not more than twonty biquadrates.

\section{Remarks :}

(1) Since 79 is not expressible as a sum of eighteen biquadrates, nineteen, if true, is best possible; but we are unable to improve the theorem further at present.

(2) All numbers upto $10^{310}$ are sums of 19 biquadrates. This is proved by Henry Thomas Jr. using extensive numerical calculation ([7], Th. 3.3). Our method yields that all numbers bigger than $10^{700}$ are sums of 19 biquadrates. to [1]

(3) For the history of the problem, we refer the reader

Our work is based upon the papers of Chen Jing run [2] and Davenport [4]. The extensivecomputer work necessary was done by Thomas ([6] and [7]) and we have freely borrowed these results. 


\section{Acknowledgement :}

The author wishes to thank Professor K. Ramachandra for his encouragement and the interest he evinced at every stage of the work. The author also wishes to thank Professor H. Halberstam for his encouragement and for the interest he showed in the work. Part of the work was done when the author was visiting the Institute For Advanced Study, Princeton, (supported in part by NSF grant MCS 77-18723 A04) and the University of Illinois, Urbana. The author is thankful to these institutions for their hospitality.

\section{§ 2. Notation :}

The following notation will be used throughout this paper.

$$
\begin{aligned}
& e(x)=e^{2 \pi i x} \\
& s_{a, q}=\sum_{x=1}^{q} e\left(\frac{a x^{4}}{q}\right) .
\end{aligned}
$$

Let $N\left(>10^{400}\right.$ ) be a given integer to be represented as a sum of twenty biquadrates.

$$
\begin{aligned}
& P=\left[N^{1 / 4}\right] ; \\
& T(\alpha)=\sum_{1<x<P} e\left(d x^{4}\right)
\end{aligned}
$$

Let $\mathrm{m}$ be an integer. (In $\$ 9$, we shali choose $\mathrm{m}=10$; In other sections, $\mathrm{m}$ can either be 9 or 10$)$. 
The singular series $\mathbf{S}(n)$

$$
=\sum_{q=1}^{\infty} \sum_{\substack{a=1 \\(a, q)=1}}^{q}\left(\frac{s, q}{q}\right)^{m} \cdot\left(-\frac{a n}{q}\right)
$$

The truncated singular series

$$
\begin{aligned}
& s_{1}(n)=\sum_{\substack{q<p^{1 / 2} \\
(a, q)=1}} \sum_{\substack{a, q \\
(a)}}\left(\frac{s_{a, q}}{q}\right)^{m} \cdot\left(-\frac{a n}{q}\right) \\
& \psi(\alpha)=\int_{0}^{p} e\left(x x^{4}\right) d x
\end{aligned}
$$

The major arc $m$ is defined by

$$
\left\{\alpha:\left|\alpha-\frac{h}{q}\right|<\frac{1}{8 q p^{3}} \text { for some } q<p^{1 / 2} \text { and }(h, q)=1\right\}
$$

The minor arc $m$ is defined by

$$
\begin{gathered}
\left\{\alpha:\left|\alpha-\frac{h}{q}\right|<\frac{1}{8 q P^{3}} \text { for some } q, p^{1 / 2}<q<8 P^{3}\right. \\
\text { and }(h, q)=1\} \\
W_{0}\left(N_{0}\right)=\int_{m}(T(\alpha))^{m} e\left(-\alpha N_{0}\right) d \alpha \\
W\left(N_{0}\right)=\int_{0}^{1}(T(\alpha))^{m} e\left(-\alpha N_{0}\right) d \alpha
\end{gathered}
$$




$$
R\left(N_{0}\right)=\int_{-\infty}^{\infty}(\psi(\alpha))^{m} \text { e }\left(-\alpha N_{0}\right) d \alpha
$$

Each section contains a proposition, which is the main result of the section. Lemmas are subsidiary results negded to prove. the proposition.

§ 3. An upper bound for $S_{q, q}$

$$
\text { We recall that } s_{a, q}=\sum_{x=1}^{q} e\left(\frac{a x^{4}}{q}\right) \text {. We define }
$$

$S(q)=\max _{a}(S, q)$.

$$
(a, q)=1
$$

In this section, we prove

Prop. 1: There holds $|\mathrm{S}(\mathrm{q})|<(4.3) \mathrm{q}^{3 / 4}$

In order to prove fhe proposition, we need

Lemma 1 (a) $\mathrm{S}(\mathrm{q})$ is a multiplicative function of $q$

(b) If $\mathrm{p} \neq 2$, and $\mathrm{a} \neq 0(\bmod \mathrm{p})$,

$$
\left|\mathrm{s}_{\mathrm{a}, \mathrm{p}}\right|<(\delta-1) \mathrm{p}^{1 / 2}
$$

where $\delta=(4, p-1)$

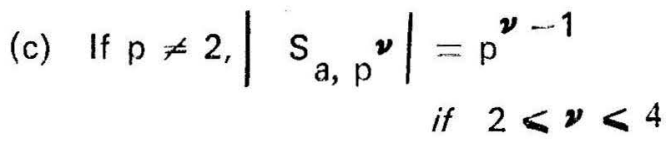

(d) If $p>2,\left|\mathrm{~s}_{a, p^{\nu}}\right|=\mathrm{p}^{3}\left|\mathrm{~S}_{\mathrm{a}, \mathrm{p}} v-4\right|$

$$
\text { if } \nu>4
$$




\section{Proof :}

A proof can be found in Davenport [3] (Lemma 6 (page 31) Lemma 12 (page 42), Lemma 13 (page 43) and Lemma 14 (page 44))

From Lemma 1, it follows that

$$
\begin{aligned}
& \frac{s(q)}{q^{3 / 4}}=\alpha_{p \| q}^{\alpha} \frac{s\left(p^{\alpha}\right)}{p^{\frac{3}{1} \alpha}} \\
& <{ }_{p} \max \left(1, \max _{\alpha>0} \frac{s\left(p^{\alpha}\right)}{p^{\frac{3}{4} \alpha}}\right)
\end{aligned}
$$

which being a finite product can be evaluated and this gives the proposition. For further details, we refer the reader to Theorem 2.1 in page 38 of Thomas [6].

\section{\$ 4. A lower bound for $R\left(N_{0}\right)$}

Let us recall that

$$
\begin{aligned}
& \psi(\alpha)=\int_{0}^{P} e\left(d x^{4}\right) d x \\
& R\left(N_{0}\right)=\int_{-\infty}^{\infty}(\psi(\alpha))^{m} e\left(-\alpha N_{0}\right) d \alpha \\
& W\left(N_{0}\right)=\int_{0}^{1}(T(\alpha))^{m} e\left(-\alpha N_{0}\right) d \alpha
\end{aligned}
$$

Define $B=B(\alpha)= \begin{cases}P & \text { if }|\alpha|<p^{-4} \\ \left.2 i \alpha\right|^{-1 / 4} & \text { if }|\alpha|>p^{-4}\end{cases}$ 


\section{Lemma 2 :}

Let $\mathrm{f}(\mathrm{x})$ be a real function which is twice differentiable in $A<x<B$; suppose that, in the interval $A \leq x<B$, we have $0<f^{\prime}(x) \leqslant$ and $f^{\prime \prime}(x)>0$. Then

$$
\underset{A<n<B}{X} e(f(n))=S_{A}^{B} e(f(x)) d x+4 \theta
$$

Proof :

This is Lemma 13 (page 34) in Vinogradov [9]

\section{Lemma 3}

We have $|\Psi(\alpha)|<B(\alpha)$

Proof :

Clearly $|\psi(\alpha)|<P$.

To prove that $|\psi(\alpha)|<2|\alpha|^{-1 / 4}$, it suffices to prove the result for $\alpha>0$. Now a change of variable $\alpha x^{4}=y$ transforms the integral to

$$
\psi(\alpha)=\frac{1}{4 x^{1 / 4}} \int_{0}^{\alpha P^{4}} \frac{e(y) d y}{y^{3 / 4}}
$$

and the result is immediate.

Lemma 4:

$$
\text { If } N_{0}-p^{3 \frac{3}{4}}<N_{1}<N_{0} \text {, then }
$$




$$
\begin{aligned}
W\left(N_{1}\right)=R\left(N_{0}\right) & +\int_{8 P^{3}}^{1-\frac{1}{8 P^{3}}}(T(\alpha))^{m} e\left(\alpha N_{1}\right) d \alpha \\
& +\theta 10^{6} p^{m-5}+3 / 4
\end{aligned}
$$

\section{Proof :}

$$
\begin{aligned}
& \text { By Lemma } 2 \text {, if }|\alpha|<\frac{1}{8 \mathrm{P}^{3}} \text {, we have } \\
& \begin{aligned}
T(\alpha) & =\underset{1<x<P}{\sum} e\left(\alpha x^{4}\right)=\int_{0}^{P} e\left(\alpha x^{4}\right) d x+4 \theta \\
& =\psi(\alpha)+4 \theta
\end{aligned}
\end{aligned}
$$

Hence by Lemma 3 ,

$$
\begin{gathered}
I(T(\alpha))^{m}-(\psi(\alpha))^{m} 1<4 m(\max (T(\alpha), \psi(\alpha)))^{m-1} \\
<4 m(B+4)^{m-1} \\
W\left(N_{1}\right)=\int_{0}^{1}(T(\alpha))^{m l} e\left(-\alpha N_{1}\right) d \alpha \\
=\int_{-\frac{1}{8 P^{3}}}^{1-\frac{1}{8 P^{3}}}(T(\alpha))^{m} e\left(-\alpha N_{1}\right) d \alpha
\end{gathered}
$$


8

$$
=\int_{|\alpha| \leq \frac{1}{8 p^{3}}} \cdots \cdots+\int_{-\frac{1}{8 p^{3}}}^{1-\frac{1}{8 p^{3}}} \cdots \cdots
$$

In the first integral, we replace $(T(\alpha))^{m}$ by $(\psi(\alpha))^{m}$ with an error $E_{1}$. Then we replace $e\left(-\alpha N_{1}\right)$ by $e\left(-\alpha N_{0}\right)$ with an error $E_{2}$. Now we extend the range of integration to $[-\infty . \infty]$ with an error $E_{3}$. Hence

$$
\begin{aligned}
& W\left(N_{1}\right)=\int_{-\infty}^{\infty}(\psi(\alpha))^{m} e\left(-\alpha N_{0}\right) d \alpha \\
& =\int_{-\frac{1}{8 P^{3}}}^{1-\frac{1}{8 P^{3}}(T(\alpha))^{m} e\left(-\alpha N_{1}\right) d \alpha+E_{1}+E_{2}+E_{3}} \\
& =R\left(N_{0}\right)+\int_{\frac{1}{8 P^{3}}(T(\alpha))^{m}}^{1-\frac{1}{8 P^{3}}\left(-\alpha N_{1}\right) d \alpha} \\
& +E_{1}+E_{2}+E_{3} .
\end{aligned}
$$

Now $\left|E_{1}\right| \leq$

$$
\begin{aligned}
& \left.\mid \int(T(\alpha))^{\mathrm{m}}-(\psi(\alpha))^{\mathrm{m}}\right) \text { e }\left(-\alpha N_{1}\right) \mathrm{d} \alpha \mid \\
& |\alpha| \leq \frac{1}{8 \mathrm{P}^{3}}
\end{aligned}
$$


9

$$
\begin{aligned}
& \leq \mid \int_{\left|\alpha \leq \frac{1}{8 P^{3}}(4 m)(B+4)^{m-1} d \alpha\right|}\left(\int_{|\alpha| \leq P^{-4}}(4 m)(P+4)^{m-1} d \alpha \mid\right. \\
& \leq \mid \\
& +\left|\frac{1}{8 P^{3}} \geqslant\right| \alpha \mid \geqslant P^{-4}(4 m)\left(2|\alpha|^{-1}+4\right)^{m-1} d \alpha \\
& \leq 10^{5} P^{m-5} \text {, since } m \text { is either } 9 \text { or } 10
\end{aligned}
$$

Similarly,

$$
\begin{aligned}
& \left|E_{2}\right| \leq\left|\quad \int(\psi(\alpha))^{m}\right| e\left(-\alpha N_{1}\right)-e\left(-\alpha N_{0}\right)|d \alpha| \\
& |\alpha|<\frac{1}{8 p^{3}} \\
& \leq \int B^{m}(2 \pi)|\alpha|\left|N_{1}-N_{0}\right| d \alpha \\
& |\alpha| \leq \frac{1}{8 \mathrm{P}^{3}} \\
& <P^{3 \frac{3}{6}}(2 \pi) \int_{|\alpha| \leqslant P^{-4}} P^{m}|\alpha| d \alpha+
\end{aligned}
$$


10

$$
\begin{aligned}
& +\mathrm{P}^{3 \frac{3}{4}}(2 \pi) \int_{|\alpha|>\mathrm{P}^{-4}}\left(2|\alpha|^{-\frac{1}{4}}\right)^{\mathrm{m}}|\alpha| d \alpha \\
& <10^{5} \mathrm{P}^{\mathrm{m}-5+\frac{3}{3}} \\
& \left|E_{3}\right|<\int_{|\alpha|>\frac{1}{8 \mathrm{P}^{3}}}|\psi(\alpha)|^{\mathrm{m}} \mathrm{d} \alpha \\
& <\int_{|\alpha|>\frac{1}{8 \mathrm{P}^{3}}} \mathrm{~B}^{\mathrm{m}} \mathrm{d} \alpha \\
& \leq 10^{5} \mathrm{P}^{\frac{3}{4} \mathrm{~m}-3}
\end{aligned}
$$

This proves the result.

Lemma 5 :

$$
\begin{aligned}
& \text { With } M=\left[\frac{P}{2}^{3 \frac{3}{4}}\right] \\
& \sum_{1 \leq A \leq M} \sum_{1 \leq B \leq M} W\left(N_{0}-A-B\right)=M^{2} R\left(N_{0}\right) \\
& +\theta 10^{7} M^{2} P^{m-5+3}
\end{aligned}
$$

Proof :

In lemma 4, we take $N_{1}=N_{0}-A-B$ and sum over $1<A<M$ and $1<B<M$. This gives 
11

$$
\begin{aligned}
& \sum_{A} \sum_{B} W\left(N_{0}-A-B\right)=M^{2} R\left(N_{0}\right) \\
& 1-\frac{1}{8 p^{3}} \\
& \left.+\int \quad(T(\alpha))^{m} e\left(-\alpha N_{0}\right) \underset{A}{(\Sigma} e(\alpha A)\right)^{2} d \alpha \\
& \frac{1}{8 \mathrm{P}^{3}} \\
& +\theta 10^{6} M^{2} p^{m-5+3}
\end{aligned}
$$

Since $\left|\sum_{A} e(\alpha A)\right| \leq \frac{1}{\|\alpha\|}$, the integral appearing on the right, is bounded, in absolute value, by

$$
\int_{\frac{1}{8 P^{3}}}^{1-\frac{1}{8 P^{3}}} P^{m}\left(\frac{1}{\|d\|}\right)^{2} d d \leq 10^{4} P^{m+3}
$$

Lemma 6

We have

$$
\begin{array}{rl}
\sum_{1 \leq A} \leq M \quad 1 \leq B \leq M & W\left(N_{0}-A-B\right) \\
& >T_{m}\left(\frac{m-1}{4}\right) M^{2} N_{0}^{\frac{m}{4}-1}
\end{array}
$$


where $\quad T_{m}=\frac{(\Gamma(5 / 4))^{m}}{\Gamma\left(1+\frac{m}{4}\right)}$

\section{Proof :}

By lemma 3 (page 22) of Vinogradov [9], the number of integer solutions $k_{r}(N)$ of $x_{1}^{4}+x_{2}^{4}+\cdots \cdots+x_{r}^{4} \leq N$ is given by

$$
\begin{aligned}
K_{r}(N) & =T_{r} N^{\frac{r}{4}-\theta r N^{r-1}} \text { where } \\
T_{r} & =\frac{(\Gamma(5 / 4))^{r}}{\Gamma\left(1+\frac{r}{4}\right)}
\end{aligned}
$$

Now,

$$
\begin{aligned}
& \sum_{1 \leq B \leq M} W\left(N_{0}-A-B\right) \\
= & \sum_{B}\left(K_{m}\left(N_{0}-A-B\right)-K_{m}\left(N_{0}-A-B-1\right)\right) \\
= & K_{m}\left(N_{0}-A-1\right)-K_{m}\left(N_{0}-A-M-1\right)
\end{aligned}
$$

$=T_{m}\left[\left(N_{0}-A-1\right)^{\frac{m}{4}}-\left(N_{0}-A-M-1\right)^{\frac{m}{4}}\right]+2 m \theta \frac{\frac{m-1}{4}}{N_{0}}$

$>T_{m} \frac{m-1}{4} M N_{0}^{\frac{m}{4}}$

and hence the lemma. 
Prop. 2

We have, for $\mathrm{m}=9$ or 10 ,

$$
R\left(N_{0}\right)>0.25 \quad N_{0}^{4}-1
$$

\section{Proof :}

From lemmas 5 and 6 , it follows that

$$
R\left(N_{0}\right)>\left(\frac{T_{m}^{(m-1)}}{4}-10^{-5}\right) N_{0}^{\frac{m}{4}-1}
$$

and hence the result.

§ 5: A lower bound for $S_{1}\left(N_{0}\right)$ :

Let us recall that $S(n)=S(n, m)$ is given by

$$
\begin{aligned}
S(n) & =\sum_{q=1}^{\infty} \sum_{a=1}^{q}\left(\frac{s, q}{q}\right)^{m} \text { e }\left(-\frac{a n}{q}\right) \\
& =\sum_{q} A(n ; q) \text { say }
\end{aligned}
$$

Write $\chi_{p}(n)=\chi_{p}(n ; m)=\sum_{i=1}^{\infty} A\left(n ; p^{i}\right)$.

\section{Lemma 7 :}

$\mathrm{A}(\mathrm{n} ; \mathrm{q})$ is a multiplicative function of $q$ and hence

$$
S\left(n_{i}=\pi_{p}\left(1+\chi_{p}(n)\right)\right.
$$




\section{Proof :}

We refer the reader to Lemma 2.11 (page 20) of Vaughan [8]

\section{Lemma 8 :}

(a) For any prime $p$, and natural number $\lambda$, let $\mathrm{N}_{\mathrm{m}}\left(\mathrm{p}{ }^{\lambda}, \mathrm{n}\right)$ denote the number of solutions of

$$
\begin{aligned}
& x_{1}^{4}+x_{2}^{4}+\cdots \cdots+x_{m}^{4}=n\left(\bmod p^{\lambda}\right), \\
& 1 \leq x_{i} \leq p^{\lambda} \text { and not all } x_{i}=0(\bmod p)
\end{aligned}
$$

Further set $\gamma=1$ if $p$ is odd and $\gamma=4$ if $p=2$. Let $\mathrm{p}^{4 \mathrm{r}+\mathrm{s}}$ exactly divide $\mathrm{n}, 0 \leq \mathrm{s} \leq 3$.

$$
\mathrm{k}_{0}=\max (4 r+s+1,4 r+\gamma) \text {. Then }
$$

$$
\begin{aligned}
& A\left(n, p^{\lambda}\right)=0 \text { for } \lambda>k_{0} \text { and } \\
& 1+x_{p}(n, m)=p^{-(m-1) \gamma} N_{m}\left(p^{\gamma}, 0\right)\left\{\sum_{\tau=0}^{r-1} p^{\tau(4-m)}\right\} \\
& +p^{(4-m) r-(m-1) \gamma} N_{m}\left(p^{\gamma}, n p^{-4 r}\right)
\end{aligned}
$$

where the empty sum is understood to be zero.

(b) Let $\mathrm{d}=$ g.c.d. of $(4, \mathrm{p}-1)$. Then for $\mathrm{p} \neq 2$,

$$
\left|N_{m}(p, n)-p^{m-1}\right| \leq\left(1-\frac{1}{p}\right)(d-1)^{m} p^{\frac{m}{2}}
$$




\section{Proof :}

For the proof of Lemma 8 (a), we refer the reader to Hilfsatz 293 of LANDAU [5] or Prop. 4. 3 of Thomas [6]. For the proof of lemma 8 (b), we refer to lemma 41 (page 91) of Thomas [6].

From Lemma 8, we have

\section{Lemma 9 :}

$$
\begin{aligned}
& \text { Let } K>100 ; \quad S_{2}=\{p>K ; p=3(\bmod 4)\} \\
& S_{4}=\{p>K ; p=1(\bmod 4)\} . \text { Then for } d=2 \text { or } 4
\end{aligned}
$$

$\operatorname{pes}_{d}^{\pi}\left(1+X_{p}(n)\right)$

$$
>\exp \left(-2(d-1)^{m} k^{1-\frac{m}{2}}\left(1+\frac{K}{2 m-8}\right)\right)
$$

\section{Lemma 10 :}

$$
\begin{aligned}
& \text { We have }{ }_{p>114}^{\pi}\left(1+X_{p} \quad(n)\right)>0.97 \\
& \text { Since } \underset{p>114}{\pi}\left(1+\chi_{p}(n)\right)
\end{aligned}
$$

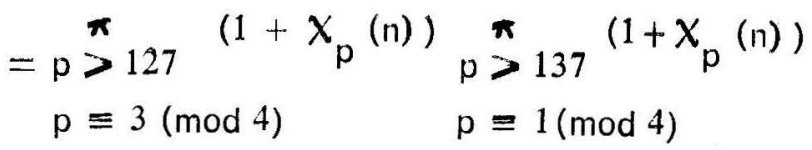

the result follows from Lemma 9.

\section{Lemma 11 :}

$$
\text { We have } \underset{2<p<113}{\pi}\left(1+X_{p}(n)\right)>0.297
$$




\section{Proof :}

This is easily verified using Lemma 8 (a). For details, we refer the reader to Thomas [6]

\section{Lemma 12 :}

$$
\begin{aligned}
& \text { If } n \equiv \tilde{n}(\bmod 16), 2<\tilde{n}<m-2 \text {, then } \\
& 1+\chi_{2}(n)=\left(\begin{array}{c}
\underset{n}{\sim}
\end{array}\right) 2^{-m+4} \text { and hence } \\
& 1+X_{2}(n)>\frac{45}{64} \\
& \text { Here }\left(\begin{array}{c}
\sim \\
\sim
\end{array}\right) \text { is the binomial coefficient }
\end{aligned}
$$

\section{Proof :}

This is straight forward. For details, we refer the reader to Theorem 4. 2 of Thomas [6].

\section{Lemma 13 :}

$$
\begin{aligned}
& \text { We have } S(n)>0.2025 \\
& \text { provided } n \equiv 2,3,4,5,6 \text { or } 7(\bmod 16)
\end{aligned}
$$

\section{Proof :}

This follows from lemmas $7,10,11$ and 12 . 
17

Lemma 14 :

We have $S(n)-S_{1}(n)<0.002$

Proof :

Since $\left|S(n)-S_{1}(n)\right|=$

$$
\begin{gathered}
\mid \sum_{q>p^{\frac{1}{2}}} \sum_{\substack{a=1 \\
(a, q)=1}}\left(\frac{s a, q}{q}\right)^{m} \text { e }\left(-\frac{a n}{q}\right) \mid \\
<\sum_{q>p^{b}} \sum_{a=1}^{q}\left(4.3 q^{-\frac{r}{4}}\right)^{m},
\end{gathered}
$$

we are through.

Prop 3 ;

There holds the inequality

$$
S_{1}(n)>0.2
$$

if $n=I(\bmod 16)$, and $/ \varepsilon(2.3,4,5,6,7)$.

\$ 6: A lower bound for $W_{0}\left(N_{0}\right)$ :

Let us recall that $\mathrm{W}_{0}\left(\mathrm{~N}_{0}\right)=$

$$
\int_{m}(T(d))^{m} \text { e }\left(-\alpha N_{0}\right) d \alpha \text {. }
$$


Lemma 15 :

$$
\begin{aligned}
& |f| \beta \mid<\frac{1}{8 q P^{3}} \text {, then } \\
& \begin{aligned}
\left(T\left(\frac{a}{q}+\beta\right)\right)^{m} & =\left(\psi(\beta) \frac{S_{a, q}}{q}\right)^{m} \\
& +\theta .4 q m\left(4.3 B(\beta) q^{-1}+4 q\right)^{m-1}
\end{aligned}
\end{aligned}
$$

\section{Proof :}

We have,

$$
\begin{aligned}
T\left(\frac{a}{q}+\beta\right) & =\sum_{x=1}^{p} e\left(\left(\frac{a}{q}+\beta\right) x^{4}\right) \\
& =\sum_{y=0}^{q-1}-\frac{y}{q}<t<\frac{p-y}{q} \\
& \left.=\sum_{y} \sum_{t} e\left(\frac{a y^{4}}{q}+\beta\right)(q t+y)^{4}\right) \\
& \left.\left.=\sum_{y} e\left(\frac{a y^{4}}{q}\right) D_{y}(z), \text { say }+y\right)^{4}\right)
\end{aligned}
$$

Now, using Lomma 2 . 


$$
\begin{aligned}
D_{Y} \quad(z)= & \int^{\frac{p-y}{q}} e\left(\beta(q t+y)^{4}\right) d t+4 \theta \\
& -\frac{y}{q} \\
= & \frac{1}{q} \int_{0}^{P} e\left(\beta x^{4}\right) d x+4 \theta \\
= & \frac{1}{q} \psi(\beta)+4 \theta .
\end{aligned}
$$

Hence $T\left(\frac{a}{q}+\beta\right)=\frac{S_{a}, q}{q} \psi(\beta)+4 \theta q$

The result follows from the following inequalities.

$$
\begin{aligned}
& \left|\frac{S_{a}, q}{q}\right|<(4.3) q^{-\frac{1}{2}} \\
& |\psi(\beta)|<B(\beta) \text { and } \\
& a^{m}-b^{m}<m(a-b) \max \left(|a|^{m-1},|b|^{m-1}\right)
\end{aligned}
$$

\section{Lemma 16 :}

\section{We have}

$$
W_{0}\left(N_{0}\right)=S_{1}\left(N_{0}\right) R\left(N_{0}\right)+\theta 10^{13} P^{m-\frac{9}{2}}
$$

\section{Proof :}

Since the proof is similar to that of Lemma 4, we give only the sketch of the proof. 


$$
\begin{aligned}
& w_{0}\left(N_{0}\right)=\int_{m}(T(\alpha))^{m} e\left(-\alpha N_{0}\right) d \alpha \\
& \frac{a}{q}+\frac{1}{8 q P^{3}} \\
& =\sum_{q<P^{\frac{1}{2}}} \sum_{(a=1}^{q} \int_{(a, q)=1} \frac{a}{q}-\frac{1}{8 q P^{3}}(T(\alpha))^{m} e\left(-N_{0} \alpha\right) d \alpha \\
& =\sum_{q<P^{\frac{1}{2}}} \sum_{a=1}^{q} \frac{1}{8 q P^{3}} \int_{(a, q)=1}-\frac{1}{8 q P^{3}}
\end{aligned}
$$

First we replace $\left(\mathrm{T}\left(\frac{\mathrm{a}}{\mathrm{q}}+\beta\right)\right)^{\mathrm{m}}$ by $\left(\psi(\beta) \frac{\mathrm{s}_{\mathrm{a}}, \mathrm{q}}{\mathrm{q}}\right)^{\mathrm{m}}$ and the error is, by Lemma 15, at most $10^{12} \mathrm{p}^{\mathrm{m}-9 / 2}$. Now we extend the range of integration of the integral to $[-\infty, \infty]$ which gives an error at most $10^{12} p^{m-9 / 2}$. Hence

$$
\begin{aligned}
w_{0}\left(N_{0}\right)= & \sum_{q<P^{\frac{1}{4}}} \sum_{\substack{a, q)=1 \\
(a, q}}^{q} \int_{-\infty}^{\infty}(\Psi(\beta)){ }^{m}\left(\frac{S_{a}, q}{q}\right)^{m} \\
& \quad \quad\left(-N_{0}\left(\frac{a}{q}+\beta\right)\right) d \beta+\theta 10^{13} P^{m-9 / 2} \\
= & S_{1}\left(N_{0}\right) R\left(N_{0}\right)+\theta 10^{13} P^{m-9 / 2}
\end{aligned}
$$


Prop 4: We have

$$
w_{0}\left(N_{0}\right)>0.05 N_{0}^{\frac{m}{4}-1}
$$

provided $N_{0}=2,3,4,5,6$ or $7(\bmod 16)$

\section{Proof :}

This follows from Prop. 2, Prop. 3 and Lemma 16.

§ 7 : The estimate on the minor arc:

We define $f(n)=n^{-a} \pi_{p / n}\left(1-p^{-a}\right)^{-1}$ where a $(>0)$ is a constant. Uitimately we shall choose $a=0.1$

$$
\begin{aligned}
& g(n)=\sqrt{f(n) ;} h(n)=\sum_{l / n} \frac{1}{g(l)} \\
& C_{a}=\sum_{p}\left(1+\frac{1}{P\left(P^{a}-1\right)}\right) \\
& D_{a}=\pi_{p}\left(1+\frac{\left(1-p^{-a}\right)^{-\frac{1}{2}}-1}{P}\right) \\
& k(m)=\sum_{d, m} \frac{1}{h(d)}
\end{aligned}
$$

\section{Lemma 17}

Let $\lambda(n)$ be a non negative multiplicative function with ${ }_{\mathrm{p}}\left(1+\frac{\lambda(\mathrm{p})}{\mathrm{p}}\right)$ convergent. Then 


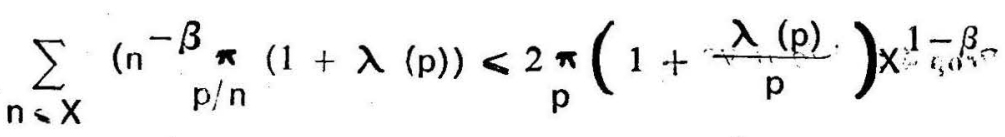

$$
\begin{aligned}
& \text { if }|\beta|<t
\end{aligned}
$$

Pro:f:

$$
\begin{aligned}
& \sum_{n \rightarrow X}\left(n^{-\beta} \sum_{p / n}^{\pi}(1+\lambda(p))=\sum_{n \leqslant x} n^{-\beta} \sum_{d / n} \mu^{2}(d) \lambda(d)\right. \\
& =\sum_{d} \mu^{2} \text { (d) } \lambda(d) \sum_{n<x} n-\beta \\
& n \equiv 0 \text { (mod d) } \\
& \leqslant 2 x^{1-\beta} \sum_{d} \frac{\mu^{2}(d) \lambda(d)}{d} \\
& <2 x^{1-\beta} \sum_{p}\left(1+\frac{\lambda(p)}{p}\right)
\end{aligned}
$$

Lemma 18

\section{We have}
(a) $\sum_{n \in X} f(n) \leqslant 2 c_{a} X^{1-a}$
(b) $\sum_{n<x} g(n) \leqslant 2 D_{a} x^{1-\frac{a}{2}}$
(c) $\sum_{n<X} g(n) n^{a / 2}<2 D_{a} x$
(d) $\sum_{n \leqslant x} \frac{1}{g(n)}<x^{1+2}$
(d) $\sum_{n \leq x} \frac{1}{f(n)}<x^{1+a}$ 


\section{Proof:}

(a), (b), and (c) follow from Lemma 17 by the proper choice of $\lambda(n)$ and $\beta$; since $\frac{1}{g(n)} \leq n^{a / 2}$ and $\frac{1}{f(n)} \leq n^{a}$, (d) and (e) follow.

Lemma 19: We have

$$
\sum_{n \leq P^{2}} f(n)(h(n))^{2} \leq 20 P^{2-a} C_{a} D_{a}^{2}(\log P)
$$

Proof :

$$
\begin{aligned}
& \sum_{n \leq p^{2}} f(n) h^{2}(n)=\sum_{n \leq p^{2}} f(n)\left(\sum_{l / n} \frac{1}{g(l)}\right)^{2} \\
& I \leq \mathrm{P}, \quad \mathrm{n} I \leq \mathrm{P} \\
& <2 \sum_{I_{1}<P} \frac{1}{g\left(I_{1}\right)} \sum_{I_{2}<I_{1}} \frac{1}{g(/ 2)} \\
& \sum_{i} f(n) \\
& \mathrm{n}<\mathrm{P} / 2 \\
& \mathrm{n} \equiv 0\left(\bmod \left[I_{1}, I_{2}\right]\right) \\
& <2 \sum_{I_{1}} \frac{1}{g\left(I_{1}\right)} \sum_{I_{2}</ /} \frac{1}{g\left(I_{2}\right)} \\
& \sum \\
& f(m) f\left(\left[/ 1, I_{2}\right]\right) \\
& \mathrm{m}<\frac{\mathrm{Fi}{ }_{2}}{\left[I_{1}, \mathrm{I}_{2}\right]}
\end{aligned}
$$


$<4 c_{a} \sum_{I_{1}} \frac{1}{g\left(I_{1}\right)} \sum_{I_{2}} \frac{f\left(\left[I_{1}, I_{2}\right]\right)}{g\left(I_{2}\right)}\left(\frac{P / 2}{\left[I_{1}, I_{2}\right]}\right)^{1-a}$

$<4 c_{a} P^{1-a} \sum_{d} \sum_{\left(I_{1}, I_{2}\right)=d} \frac{1}{g\left(I_{1}\right)}$

$$
\frac{g^{2} \frac{(/ / 2)}{d}}{g\left(I_{2}\right)}\left(\frac{d}{I_{1}}\right)^{1-a}
$$

$<4 C_{a} P^{1-a} \sum_{d} \sum_{(/ / 1, / 2)=d} \frac{1}{g(/ / 1) g(/ 2)}$

$$
\left(\frac{g\left(I_{1}\right) g\left(I_{2}\right)}{g(d)}\right)^{2} \frac{d^{1-a}}{/_{1}^{1-a}}
$$

$<4 c_{a} p^{1-a} \sum_{d} \frac{d^{1-a}}{g^{2}(d)} \sum_{1}=0(\bmod d) \frac{g\left(t_{1}\right)}{/_{1}^{1-a}}$

$$
\sum_{I_{2} \equiv 0(\bmod d)} g\left(I_{2}\right)
$$

$<8 D_{a} P^{1-a} \sum_{d} \frac{1}{d^{a / 2} g(d)} \sum_{\substack{1 \\ l}} g\left(l_{1}\right) l_{1}^{a / 2}$

$$
I_{1}=0(\bmod d)
$$

$<16 C_{a} D_{a}^{2} P^{2-a} \sum_{d} \frac{1}{d}$ 


$$
<20 C_{a} D_{a}^{2} P^{2-a}(\log P)
$$

Lemma 20 :

$$
\begin{aligned}
& \text { If } f(x)=\alpha_{k} x^{k}+\alpha_{k-1} x^{k-1}+\ldots \ldots \alpha_{0} \text { and } \\
& g_{1}(x)=f(x+l)-f(x)=\mid k \alpha_{k} x^{k-1}+\ldots, \\
& \text { then }\left|\sum_{x=1}^{P} e(f(x))\right|_{l=1}^{2}\left|\sum_{x=1}^{P-1} e\left(g_{j}(x)\right)\right|+P
\end{aligned}
$$

Proof :

$$
\begin{aligned}
& \left|\sum_{x=1}^{P} e(f(x))\right|^{2}=\sum_{x} \sum_{y} e(f(y)-f(x)) \\
& <\left|\sum_{\substack{x \\
x=y}} \sum_{y} e(f(y)-f(x))\right| \\
& +2\left|\sum_{\substack{x \\
y>x}} \sum_{y} e(f(y)-f(x))\right|
\end{aligned}
$$

We write $y=x+l$ in the second sum and simplify. This gives the result.

\section{Lemma 21:}

$$
\text { If }\left|x-\frac{\mathrm{h}}{\mathrm{q}}\right|<\frac{1}{8 \mathrm{q} \mathrm{P}^{3}} \text { and } \mathrm{P}<\mathrm{q}<8 \mathrm{P}^{3} \text {, then }
$$


$|T(\alpha)|<4 C_{a}^{1 / 8} D_{a}^{1 / 2} P^{\frac{7+a}{8}}(\log P)^{\frac{1}{4}}$

\section{Proof :}

By Lemma 20,

$$
|T(\alpha)|^{2}<2 \sum_{I=1}^{P}\left|\sum_{x=1}^{P-1} \mathrm{e}\left(4 \alpha / x^{3}+\ldots\right)\right|+P
$$

Hence

$$
\begin{aligned}
& T_{1}(\alpha)=|T(\alpha)|^{2}-P \\
& <2 \sum_{I=1}^{P}\left|\sum_{x=1}^{P-I} e\left(4 \alpha / x^{3}+\cdots\right)\right| \\
& \left|T_{1}(\alpha)\right|^{2}<4\left(\sum_{l=1}^{P} g(l)\right)\left(\sum_{l=1}^{P} \frac{1}{g(l)}\right. \\
& \left.\left|\sum_{x} e\left(4 d / x^{3}+\ldots\right)\right|^{2}\right) \\
& <8 D_{a} P^{1-\frac{a}{2}}, \sum_{l=1}^{P} \frac{1}{g(I)} \\
& \left|\sum_{x} e\left(4 d / x^{3}+\ldots\right)\right|^{2},
\end{aligned}
$$

By Lemma 20 and 19 (d), 


$$
\left.\begin{array}{rl}
T_{2}(\alpha)= & \frac{\left|T_{1}(\alpha)\right|^{2}}{8 D_{a} P}<\sum_{I=1}^{P} \frac{1}{g(I)} \\
<2 & \left\{\sum_{I=1}^{P}\left|\sum_{x=1}^{P-I} \mathrm{e}\left(12 \alpha / I x^{2}+\ldots\right)\right|+P\right\}
\end{array}\right\}
$$

$$
\begin{aligned}
& \mathrm{T}_{3}(\alpha)=\mathrm{T}_{2}(\alpha)-\mathrm{p}^{2+\mathrm{a}}<2 \sum_{I, I_{1}} \frac{1}{\mathrm{~g}(I)} \\
& \mid \sum_{x=1}^{P-I-I_{1}} \text { e }\left(12 \alpha / I_{1} x^{2}+\ldots \ldots\right) \mid \\
& <2 \quad \sum h(n) \\
& n<\frac{\mathrm{P}^{2}}{4} \\
& P-I-I_{1} \\
& \max _{1}\left|\sum_{x=1} \mathrm{e}\left(12 \alpha / /_{1} x^{2}+\ldots \ldots\right)\right|
\end{aligned}
$$




$$
\begin{aligned}
& \left|T_{3}(\alpha)\right|^{2}<4\left(\sum_{n} f(n) h^{2}(n)\right)\left(\sum_{n} \frac{1}{f(n)}\right. \\
& \left.I_{1}^{\max }=\mathrm{n}\left|\sum_{\mathrm{x}} \mathrm{e}\left(12 \alpha \|_{1} \mathrm{x}^{2}+\ldots . .\right)\right|^{2}\right) \\
& \left.<\frac{\left(80 P^{2}-a\right.}{2^{2-a}} C_{a} D_{a}^{2} \log P\right)\left(\sum_{n} \frac{1}{f(n)}\right. \\
& \max _{l}\left|\sum_{1=n} \mathrm{e}\left(12 \alpha / I_{1} x^{2}+\ldots \ldots\right)\right|^{2} \\
& T_{4}(\alpha)=\frac{2^{2-a} T_{3}(\alpha)^{2}}{80 P^{2-a} C_{a} D_{a}^{2} \log P}<\sum_{n} \frac{1}{f(n)} \\
& \max _{1=n}\left|\sum_{x} e\left(12 \alpha I_{1} x^{2}+\ldots \ldots\right)\right|^{2} \\
& <\sum_{n} \frac{1}{f(n)} \max _{I I_{1}=n}\left\{\begin{array}{c}
P-/-I_{1} \\
2 \sum_{I_{2}=1}
\end{array}\right. \\
& \left.\left|\sum_{x=1}^{P-I-I_{1}-I_{2}} \mathrm{e}\left(24 \alpha / I_{1} I_{2} x+\ldots \ldots\right)\right|+P\right\} \\
& <2 \sum_{n} \frac{1}{f(n)} \\
& \max _{I} \sum_{1=n}^{P-I-I} \min \left(P, \frac{1}{2 ! 24 \alpha I_{1} I_{2} I^{\prime !}}\right)+P^{3+2 a}
\end{aligned}
$$




$$
<2 \sum_{m} k(m) \cdot \min \left(P,-\frac{1}{2 \mid 24 \alpha m \|}\right)+p^{3+2 a}
$$

One easily checks thaf $k(m)<m^{a}$ and $m=1 / I_{2}<\frac{p^{3}}{27}$;

We also have $a=0.1$. Hence

$$
T_{4}(\alpha)<2 \sum_{m<\frac{P^{3}}{27}}^{a} \min \left(P, \frac{1}{2 \mid 24 d m \|}\right)+P^{3+2 a}
$$

$<2\left(\frac{P^{3}}{27}\right)^{a} \sum \min \left(P, \frac{1}{2\|24 ; m\|}\right)+P^{3+2 a}$

$$
m<\frac{p^{3}}{27}
$$

For a given $\alpha$, we know that there existh and $q,(h, q)=1$ such that $\left|\alpha-\frac{h}{q}\right| \leqslant \frac{1}{8 a p^{3}}, q<8 P^{3}$.

Let $\mathrm{q}^{\prime}=\frac{q}{(24, \mathrm{q})}$. The $\mathrm{m}$-sum is divided into at most $\left(\frac{p^{3}}{27 q^{\prime}}+1\right)$ subintirvals, each of length $q^{\prime}$. In each subinterval, wo check that the sum is atmost $2 P+q^{\prime} \log q^{\prime}+q^{\prime}$.

Hence

$$
\begin{aligned}
\left|T_{4}(\alpha)\right| & <2 \frac{p^{0.3}}{(27)^{0.3}}\left(\frac{p^{3}}{27 q^{\prime}}+1\right) \\
& \left(2 p+q^{\prime} \log q^{\prime}+q^{\prime}\right)+p^{3+2 a} \\
& <25 p^{3.3} \log p
\end{aligned}
$$


Hence $\left|T_{3}(\alpha)\right|^{2}<10^{3} \mathrm{P}^{5.2} \mathrm{C}_{\mathrm{a}} \mathrm{D}_{\mathrm{a}}^{2}(\log \mathrm{P})^{2}$

Hence $\left|T_{2}(\alpha)\right|<(31.63) \mathrm{P}^{2.6} \mathrm{C}_{\mathrm{a}}^{\frac{1}{2}} \mathrm{D}_{\mathrm{a}}(\log \mathrm{P})$

Hence $\left|T_{1}(\alpha)\right|<(15.91) P^{\frac{7.1}{4}} C_{a}^{\frac{1}{4}} D_{a}(\log P)^{1}$ which proves the result.

\section{Lemma 22 !}

$$
\begin{gathered}
\text { If }\left|\alpha-\frac{\mathrm{h}}{\mathrm{q}}\right|<\frac{1}{8 \mathrm{qP}^{3}} \text { and } \mathrm{P}^{\mathrm{t}}<\mathrm{q}<\mathrm{P}, \\
\text { then }|\mathrm{T}(\alpha)|<100 \mathrm{P}^{7 / 8} .
\end{gathered}
$$

\section{Proof :}

Arguing as in Lemma 9.5 of Thomas [6] or Lemma 9 of Davenport [3], we have the result. (Lemma 9.5 of Thomas [6] is proved under the restriction $\left|\alpha-\frac{h}{q}\right|<\frac{1}{64 \mathrm{qP}^{3}}$ but the same proof holds in our case).

\section{Proof :}

$$
\text { On the minor are }|T(\alpha)|<40 \mathrm{P}^{\frac{71}{8}}(\log P)^{\frac{1}{6}}
$$

Proof :

The proposition foliows from Lemmas $\mathbf{2 1}$ and 22 
\$ 8 : A lower bound for the number of integers upto $X$ representable as a sam of five fourth powers

\section{Lemma 23 :}

Let $P \geqslant 10^{10}$. Let $\mathrm{M}$ be the number of solutions of the equation $\mathrm{x}^{4}+\mathrm{u}_{\mathrm{h}}=\mathrm{y}^{4}+\mathrm{u}_{\mathrm{j}}$, subject to (i) $\mathrm{P}<\mathrm{x}, \mathrm{y}<2 \mathrm{P}$ (ii) $x \equiv y(\bmod 2)($ iii $) u_{h}, u_{j}<P^{3}+\mu$ and (iv) $u_{h}, u_{j} \varepsilon \mathcal{~} \mathcal{~}$. Then

$$
M<P U+P^{\frac{1+3 \mu}{4}} U^{5 / 4} \text { B. }
$$

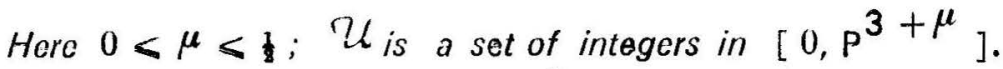
$\mathrm{U}$ is the number of elements of $\mathcal{U}$.

$$
\begin{gathered}
B^{4}=\frac{1}{\max } d_{4}(m) \\
M \leqslant \frac{P^{3+\mu}}{192}
\end{gathered}
$$

\section{Proof :}

Even though this has not been explicitly stated anywhere, this is essentially contained in Lemma 1 of Davenport [ 4 ] and Lemma 7.1 of Thomas [6].

Lemma 24 : The number of solutions of the equation

$$
a^{4}+b^{4}=x^{4}+y^{4}, \text { with } a, b, x, y<A \text {; }
$$

$a=x(\bmod 2) ; b=y(\bmod 2)$ is atmost $A^{2}(\log A)^{4}$ provided $\mathrm{A}>10^{10}$. 


\section{Proof :}

We write $x+a=2 k ; \quad x-a=2 l ; \quad y+b=2 m$ $y-b=2 n$.

Then we have to find the number of solutions of the equation $k /\left(k^{2}+l^{2}\right)=m\left(m^{2}+n^{2}\right)$, with $k, m<A ; l, n<A / 2$. The number of solutions are

$$
=\sum_{k, 1} \sum_{m, n} 1
$$

$$
m n\left(m^{2}+n^{2}\right)=k /\left(k^{2}+l^{2}\right)
$$

$=\sum_{k, l} \mathrm{~d}\left(\mathrm{k} /\left(\mathrm{k}^{2}+l^{2}\right)\right)$

$$
<\sum \frac{d^{2}(k)+d^{2}(l)}{2} d\left(k^{2}+I^{2}\right)
$$

$<\sum_{k<A} d^{2}(k) d\left(k^{2}+l^{2}\right)$

$$
\leqslant 2 \sum_{k \leqslant A} d^{2}(k) \sum_{\substack{j / k^{2}+l^{2} \\ j<2 A}} 1
$$

$<2 \sum_{k<A} d^{2}(k) \sum_{j<2 A} \sum_{k^{2}+l^{2} \equiv 0(\bmod j)}^{1}$

$<2 \sum_{k<A} d^{2}(k) \sum_{j<2 A} P(j)\left(\frac{A}{j}+1\right)$ 
where $P(j)$ is the number of solutions of $k^{2}+l^{2}=0(\bmod j)$, $1</ \leqslant j$, for a fixed $k$. We observe that $P(j)<\quad X(d)$, $\mathrm{d} / \mathrm{j}$

where $X$ is the Dirichlet character $(\bmod 4)$ and hence we easily check that

$$
\begin{aligned}
& \sum_{j<2 A} \frac{P(j)}{j}<\log A \\
& \sum_{j<2 A} P(j)<4 A
\end{aligned}
$$

and $\sum_{k<A} d^{2}(k)<\frac{A}{3} \log ^{3}(A+2)$

This proves the result.

\section{Lemma 25:}

Let I be 0,1 or 2 . The number of integers upto $X$, representable as a sum of two biquadrates and belonging to $I(\bmod 16)$ is atleast $x^{0.45}$ provided $x>10^{188}$.

\section{Proof:}

Choose $f_{0}$ and $f_{1} \varepsilon(0,1)$ such that $f_{0}+f_{1}=1$. Let $r(m)$ be the number of solutions of $m=x^{4}+y^{4}$,

$$
\begin{gathered}
x, y<\left(\frac{x}{2}\right)^{\frac{1}{t}} ; x=f_{0}(\bmod 2) ; y=f_{1}(\bmod 2) \\
\text { Tilen } \sum r(\operatorname{mi})=\left(\left(\frac{x}{2}\right)^{\frac{1}{2}} \frac{1}{2}-1\right)^{2}
\end{gathered}
$$


By lemma $24, \sum r^{2}(m)<\left(\frac{x}{2}\right)^{\frac{1}{2}}\left(\log x^{\frac{1}{4}}\right)^{4}$

$$
<x^{0.5424} \text {. }
$$

Hence the required estimate

$$
>\sum_{r(m) \neq 0} 1 \geqslant \frac{\left(\sum r(m)\right)^{2}}{\sum r^{2}(m)}
$$

and we are through.

\section{Lemma 26 :}

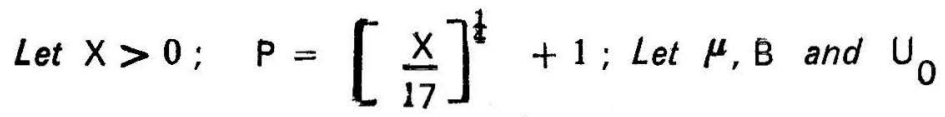

satisfy the following conditions

(i) $0<\mu<$ i

(ii) $\mathrm{B}^{4}=1 . \quad \max$

$$
m<\frac{1}{192} \mathrm{P}^{3+\mu} \mathrm{d}_{4}(\mathrm{~m})
$$

(iii) The number of integers upto $\mathrm{P}^{3}+\mu$ which are sums of ! biquadrates and belonging to $f_{0}(\bmod 16)$ is atleast $\mathrm{U}_{0}$ for every $\mathrm{f}_{0} \varepsilon(0,1,2, \ldots \ldots /)$

$$
\text { (iv) } \mathrm{B}^{4}<\mathrm{U}_{0} \mathrm{P}^{3-3 \mu} \text {. }
$$

Then the number of integers upto $X$ which are sum of $(I+1)$ biquadrates and belonging to $f(\bmod 16)$ is at/east $\frac{\mathrm{PU}_{0}}{8}$ for every $f \&(0,1,2, \ldots \ldots(1+1))$. 


\section{Proof :}

Let $f_{0} \varepsilon(0,1,2, \ldots \ldots l)$ and $f_{1} \varepsilon(0,1)$ such that $\mathrm{f}_{0}+\mathrm{f}_{1}=\mathrm{f}$. Let $\mathcal{U}=\left\{\mathrm{n}<\mathrm{P}^{3+\mu}: \mathrm{n}\right.$ is sum of $/$ biquadrates and $\left.\mathrm{n} \equiv \mathrm{f}_{0}(\bmod 16)\right\} ; U$ be the number of elements of $\mathcal{U}$; By (iii), $U>U_{0}$.

Let $r(m)$ be the number of solutions of $m=x^{4}+u_{h^{\prime}}$ $P<x<2 P ; \quad x \equiv f_{1}(\bmod 2)$ and $u_{h} \in \mathcal{U}$. The number $N$ of integers upto $X$, which are rapresentable as a sum of $(I+1)$ biquadrates and $\equiv f(\bmod 16)$ is atleast

$$
\begin{aligned}
& \sum_{m} 1 \\
& r(m) \neq 0 \\
& >\left(\sum r(m)\right)^{2} /\left(\sum r^{2} \cdot(m)\right)
\end{aligned}
$$

Now, using lemma 23

$\mathrm{N}>\left(\frac{\mathrm{PU}}{2}\right)^{2} / \mathrm{PU}+\mathrm{P} \frac{1+3 \mu}{4} \cdot \mathrm{U}^{5 / 4} \mathrm{~B}$

$>\left(\frac{\mathrm{PU}_{0}}{2}\right)^{2} / \mathrm{PU}_{0}+\mathrm{P}^{\frac{1+3 \mu}{4}} \mathrm{U}_{0}^{5 / 4} \mathrm{~B}$.

By (iv), $\mathrm{P}^{\frac{1+3 \mu}{4}} \mathrm{U}_{0}^{5 / 4} \mathrm{~B}<\mathrm{PU}_{0}$.

Hence $N>\left(\frac{\mathrm{PU}_{0}}{2}\right)^{2} / 2 \mathrm{PU}_{0}:>\frac{\mathrm{PU}_{0}}{8}$. 


\section{Lemma 27 :}

Let $f$ be $0,1,2$, or 3 . Then the number of integers upto $X$, representable as a sum of three biquadrates belonging to $f(\bmod 16)$ is at/east $x^{0.603}$ provided $x>10^{236}$.

\section{Proof :}

$$
\text { We choose } I=2 ; \mu=0.20096 \text { and } U_{0}=\mathrm{p}^{(3+\mu)(0.45)}
$$
in lemma 26 ; since $B^{4}<10^{12} \mathrm{p}^{(3+\mu)(0.235)}$, we verify (iv) in lemma 26 . This gives that the required estimate

$$
>\mathrm{PU}_{0}>\frac{1}{8}\left(\frac{\mathrm{x}}{17}\right)^{0.610108}>\mathrm{x}^{0.603}
$$

\section{Lemma 28 :}

Let $\mathrm{f}$ be $0,1,2,3$ or 4 . Then the number of integers upto $\mathrm{X}$, representable as a sum of 4 biquadrates and belonging to $f(\bmod 16)$ is at/east $x^{0.7095}$ provided $X>10^{307}$

\section{Proof :}

We choose $I=3 ; \mu=0.0869 ; U_{0}=\mathrm{p}^{(3+\mu)(0.603)}$ in lemma 26. We have to use $\mathrm{B}^{4}<10^{14} \mathrm{p}^{(3+\mu)(0.225)}$ This yields the result.

\section{Prop 61}

Let $\mathrm{f}$ be $0,1,2,3,4$ or 5 . Then the number of integers upto $X$ representable as a sum of 5 biquadrates and balonaing to $f(\bmod 16)$ is atleast $x^{0.7795}$ provided $X>10^{410}$ 


\section{Proof :}

We take $I=4 ; \mu=0.01133 ;$ and $U_{0}=P^{(3+\mu)(0.7095)}$. We use $B^{4}<(15) \times 10^{23}$. This gives the result.

\section{§. Conclusion of the proof :}

\section{I emma 30 :}

If $\mathrm{N}>10^{412}$, then $\mathrm{N}$ can be written as a sum of at most twenty hiquadrates.

\section{Proof :}

Choose $f \boldsymbol{\varepsilon}(0,1,2,3,4,5)$ and $I \varepsilon(2,3,4,5,6,7)$ such that

$$
\begin{aligned}
& N-2 f=/(\bmod 16) \\
& P=\left[N^{\frac{1}{2}}\right]
\end{aligned}
$$

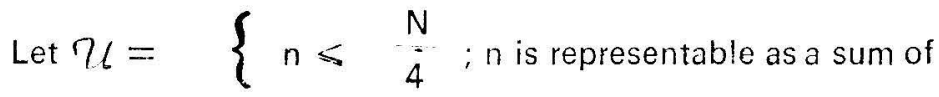
five hiquadratas: $n=f(m) d 16)\}$

$$
\begin{aligned}
& \text { Let } U(d)-u_{u}^{\Sigma} 7 l^{\circ(11 \alpha)} \\
& r(N)=\int_{0}^{1}(T(x))^{10},(u(x))^{2} \text { e }(-i v \dot{x}) \mathrm{d} x
\end{aligned}
$$




\section{8}

It suffices to prove that $r(N)>0$

$$
\text { Now r }(\mathrm{N})=\underset{m}{\boldsymbol{S}}+\underset{\boldsymbol{m}}{\boldsymbol{\rho}}
$$

Now, using Proposition 4,

$$
\begin{aligned}
\int_{m} & =\sum_{u_{1}, u_{2}} \int_{m}(T(d))^{10} e\left(-\left(N-u_{1}-u_{2}\right) d\right) d d \\
& =\Sigma W_{0}\left(N-u_{1}-u_{2}\right) \\
& u_{1}, u_{2} \\
> & 0.01 U^{2} P^{6}
\end{aligned}
$$

7 the other hand, using Proposition 5 and 6 ,

$$
\begin{aligned}
& \left.\left.\mid \int_{m}(T(\alpha))^{10}(U(\alpha))^{2} e(-N \alpha)\right)\right) d x \mid \\
& \left.<\max _{\alpha \varepsilon m} T(\alpha)\right)\left.\left|\int^{10}\right| u(\alpha)\right|^{2} d \alpha \\
& <(40)^{10} P^{\frac{71}{8}} \cdot(\log P)^{2.5} U \\
& <(40)^{10} P^{\frac{71}{8}} \cdot(\log P)^{2.5} U^{2} /\left(\frac{N}{4}\right)^{07795} \\
& \leqslant 4^{11} 10^{10} P^{5.757} U^{2}(\log P)^{2.5}
\end{aligned}
$$


It suffices to to verify that, for $P \leqslant 10^{102}$,

$4^{11} 10^{10} P^{5.757}(\log P)^{25}<0.01 P^{6}$

and this is true.

Lemma : 31

If $\mathrm{N}<10^{412}$, then $\mathrm{N}$ can be written as a sum of atmost 20 biquadrates.

\section{Proof :}

If $N \leqslant 10^{412}$, then $N-\left[N^{\frac{1}{4}}\right]^{4}<10^{310}$ and hence representable as a sum of atmost 19 biquadrates by Th. 3.3 of Thomas [7]

Thus the proof of the theorem is complete.

Note :

Some doubts have been raised about the correctness of lemma 31 and the author has not verified the calculations given in [7]. But even a weaker result, compared to lemma 31, will suffice for our purpose and we shall return to this subject at a later date. 


\section{References}

1. R. Balasubramanian, On Waring's problem:, g (4) $<21$, Hardy Ramanujan Journal, Vol. 2 (1979) (1-31).

2. (hen jing run, Waring's problem for $g(5)=37$

Chinese Mathematic Acta 6 (1965) (105-127)

3. H. Davenport, Analytic Methods for Diophantine equations and Diophantine inequalities, Ann. Arbor, Ann. Arbor Publishers, MICHGAN (1962).

4. H. Daveuport, On Waring's problem for fourth powers, Annals of Mathematics, 40 (1939), (731-747)

5. I ardau, E. Vor llcsungen uber zablen theorie, Part 6 , Das Waringshe problem, New York, Chelsea, 1955.

6. Thomas. H. E., A numerical approach to Waring's problem for fourth powers, Dissertation, Univ. of Michigan, Ann. Arbor, Michigan 1973.

7. Thomas. H.E., Waring's problem for twenty two biquadrates, Transactions of the American Math. Society, 193 (1974), (427-430).

8. R. C. Vaughan, The Hardy-Littlewood Method, Cambridye University Press, CAMBR(DG)E (1954)

9. I. M. Vinogradov, The method of trigonometrical sums in the theory of numbers, translated from the Russian, revised and annotated by Davenport. A. and Rcth K. F.; New York, Inter science ( 1954 ).

School of Mathematics,

Tata Institute of Fundamental Research,

Homi Bhabha Road.

Bombay-400005. (India) 